\title{
Gecko-inspired chitosan adhesive for tissue repair
}

\author{
Samuel J Frost ${ }^{1}$, Damia Mawad ${ }^{2,3}$, Michael J Higgins ${ }^{4}$, Herleen Ruprai ${ }^{1}$, Rhiannon Kuchel ${ }^{5}$, Richard D Tilley ${ }^{5}$, \\ Simon Myers ${ }^{1,6}$, James M Hook ${ }^{5}$ and Antonio Lauto ${ }^{1,6,7}$
}

The advent of nanotechnology has opened the possibility of fabricating nanoscopic pillars on the surface of polymeric films mimicking the Gecko's foot, in an attempt to increase their adhesive capabilities enhanced by van der Waals forces. However, these forces are considerably weakened in a wet physiological environment. To circumvent this loss in force, current biocompatible adhesives with nanopillars require complex multiple-step fabrication, including an extra layer of adhesive coating to stabilize tissue bonding under physiological conditions. In this report, we describe a simple one-step fabrication process of a single-layer chitosan film that has pillars with base diameter in the range of 100-600 nm and a height of $\sim 70 \mathrm{~nm}$. The nanostructured adhesive is laser-bonded to tissue and does not require pillar coating to enhance bonding in water. In comparison with a 'flat' adhesive (without pillars), the nanostructured adhesive bonded significantly stronger to tissue under either stress or pressure. Atomic force spectroscopy also confirmed the superior bonding capability of the nanostructured adhesive. This study demonstrates a one-step fabrication technique to produce a monolayer gecko-inspired adhesive that is biocompatible and bonds effectively to tissue.

NPG Asia Materials (2016) 8, e280; doi:10.1038/am.2016.73; published online 17 June 2016

\section{INTRODUCTION}

Since the adhesion mechanism of the Gecko's foot has been described, ${ }^{1}$ researchers have designed and fabricated adhesive films mimicking the lizard's toes, which contain arrays of keratin fibrils (setae) $\sim 100 \mu \mathrm{m}$ long and $\sim 5 \mu \mathrm{m}$ wide with a very high density $\left(5000 \mathrm{~mm}^{-2}\right)$. Setae terminate in numerous nanosized branches named spatulae with a diameter ranging between 200 and $400 \mathrm{~nm}$. When the gecko's foot comes into contact with a surface, the lizard pushes the setae towards the surface and pulls them along it for a few micrometers to increase the number of spatulae contacting the surface. As a result, van der Waals forces attract the spatula to the surface as long as the contact is preserved since these forces decrease sharply with distance. ${ }^{2}$ Gecko-inspired adhesives have arrays of nanostructures on the surface to exploit van der Waals attractive forces; $\mathrm{Qu}$ et al. $^{3}$ demonstrated the strongest adhesion to a dry surface using hierarchical carbon nanotube arrays $(1000 \mathrm{kPa})$ that was a 10 -fold increase compared with the gecko in its natural state. Despite the ability of gecko-inspired adhesives to bond strongly to dry surfaces, under wet conditions, as in wound repair, the bonding weakens significantly since a water layer can intervene between nanopillars and surface reducing the strength of van der Waals forces. ${ }^{4}$ Vajpayee et al. ${ }^{5}$ showed, for example, that a nanostructured film made of polydimethylsiloxane adhered poorly to a hydrophilic surface under water $\left(\sim 1 \times 10^{-3} \mathrm{~N}\right)$. To overcome this problem, biomedical adhesives have been developed using a multilayer system, where the interfacial area is coated so as to better adhere in a wet environment. In particular, nanopillars have been coated with a variety of compounds, including dopamine, ${ }^{6}$ oxidized dextran ${ }^{7}$ and medical-grade cyanoacrylate, ${ }^{8}$ to promote tissue crosslinking and ensuring a non-reversible chemical bond under wet physiological conditions. If coating allows for stronger adhesion, it also requires an extra fabrication step, complicating the manufacturing procedure. We herein describe a one-step fabrication approach of a nanostructured adhesive with demonstrated adhesion under wet conditions.

Gecko-inspired adhesives have the advantage of sealing wounds and repair tissue without sutures or staples, which are invasive devices. These adhesives are minimally invasive to the host tissue and can potentially provide stronger, faster and easier wound closure than conventional methods. ${ }^{9,10}$ Sutures and staples are currently the standard tools for surgeons even if their use can have adverse effects including air and fluid leakage in procedures involving lungs, ${ }^{11,12}$ blood vessels, ${ }^{13}$ dura mater, ${ }^{14}$ and urethra. ${ }^{15}$ The kidney and liver parenchymal tissue can also be difficult to suture with risk of high blood loss. ${ }^{16}$ Our group has recently developed a biocompatible and sutureless adhesive for peripheral nerve repair; ${ }^{17}$ this adhesive is based on chitosan and is bonded photochemically to tissue by a green laser without causing thermal damage. ${ }^{18,19}$ In this study, we succeeded in modifying the adhesive surface with nanopillars to further enhance the

\footnotetext{
${ }^{1}$ School of Science and Health, Western Sydney University, Penrith, NSW, Australia; ${ }^{2}$ Department of Materials, Department of Bioengineering and Institute for Biomedical Engineering, Imperial College London, London, UK; ${ }^{3}$ School of Materials Science and Engineering, University of New South Wales, Sydney, NSW, Australia; ${ }^{4}$ ARC Centre of Excellence for Electromaterials Science, Intelligent Polymer Research Institute, AllM Faculty, Innovation Campus, University of Wollongong, Wollongong, NSW, Australia; ${ }^{5}$ Mark Wainwright Analytical Centre, University of New South Wales, Sydney, NSW, Australia; ${ }^{6}$ School of Medicine, Western Sydney University, Penrith, NSW, Australia and ${ }^{7}$ Biomedical Engineering \& Neuroscience Research Group, The MARCS Institute, Western Sydney Universityy, Penrith, NSW, Australia

Correspondence: Dr A Lauto, School of Science and Health, Western Sydney University, Narellan Road, Locked Bag, Penrith South DC, Penrith, NSW 1797, Australia.

E-mail: a.lauto@westernsydney.edu.au

Received 27 November 2015; revised 16 February 2016; accepted 22 March 2016
} 
strength of tissue bonding. A simple dry-casting technique is required to fabricate the nanostructured film and no coating is necessary to stabilize the bonding between adhesive and tissue in wet environment; this feature simplifies the fabrication process to a single step procedure.

\section{MATERIALS AND METHODS}

\section{Adhesive fabrication}

The adhesive solution was prepared accordingly to the method previously published by our group. ${ }^{17,20}$ Briefly, medium molecular weight chitosan (598 cps viscosity, 81\% deacetylation; Sigma-Aldrich, Sydney, NSW, Australia) was dissolved at a concentration of $1.7 \% \mathrm{w} / \mathrm{v}$ in deionized water containing $2 \%$ $(\mathrm{v} / \mathrm{v})$ acetic acid and $0.01 \%(\mathrm{w} / \mathrm{v})$ rose bengal. The viscous solution was stirred for 14 days at room temperature $\left(\sim 25^{\circ} \mathrm{C}\right)$ in the dark to avoid photo-bleaching of the rose bengal. The homogenized rose bengal -chitosan solution was then centrifuged to remove insoluble matter at $3270 \times g$ for an hour. The supernatant was collected and spread evenly $\left(\sim 2 \mathrm{ml}\right.$ over $\left.\sim 12 \mathrm{~cm}^{2}\right)$ over either a sterile and dry polycarbonate plate (master) that had arrays of nanosize holes ranging from 100 to $600 \mathrm{~nm}$, or a flat Perspex (PMMA) plate without holes. The solution dried up losing $~ 90 \%$ of the water content and becoming a thin film, which was insoluble in water and did not swell macroscopically. ${ }^{20,21}$ The film preparation took typically 3 weeks under clean conditions, atmospheric pressure and temperature of $25^{\circ} \mathrm{C}$. The rose bengal -chitosan film was then carefully detached from the plate without damage; it measured $4 \times 3 \mathrm{~cm}$ and had a thickness of $28 \pm 3 \mu \mathrm{m}$. The adhesive was placed in a sterile plastic box to preserve the shape and stored in the dark at room temperature to avoid dye photobleaching; smaller strips were later cut with scissors for the planned experiments. The polycarbonate plate was fabricated using an injectionmoulding technique that allowed the molten polycarbonate to fill a mould cavity where the mirror-image stamper of the plate was placed. ${ }^{22,23}$ The polycarbonate plate was then retrieved and used for the experiments after quality inspection.

\section{Photochemical tissue bonding}

The chitosan adhesive was tested in vitro on calf intestine, tissue sections $\left(\sim 20 \times 10 \mathrm{~mm}^{2}\right)$ were bisected and the adventitial layer gently removed with scissors under an Olympus operating microscope (Olympus, Macquarie Park, NSW, Australia). The intestine was kept moist using $\sim 40 \mu \mathrm{l}$ of water while the incision stumps were approximated end-to-end, excess water was removed with cotton tips before an adhesive film $\left(10 \times 6 \mathrm{~mm}^{2}\right)$ was positioned across the bisection line on the serosa layer with microforceps ensuring full contact with the intestine. Thereupon, a green laser serially spot-irradiated $(\sim 0.5 \mathrm{~cm})$ the strip ensuring each spot was irradiated for $\sim 5 \mathrm{~s}$ before moving the beam to the adjacent spot. The laser beam scanned the whole surface area of the adhesive several times in order to deliver $\sim 110 \mathrm{~J} \mathrm{~cm}^{-2}, 19$ during irradiation the adhesive temperature remained below $39^{\circ} \mathrm{C} \cdot 17,18$ The laser was coupled to a multimode optical fiber (core diameter $=200 \mu \mathrm{m}$ and numerical aperture $=0.22$ ) that was inserted into a hand-held probe to provide easy and precise beam delivery. The laser parameters are summarized in Table 1.

\section{Repair strength}

The repair strength of samples was tested with a calibrated single-column tensiometer (Instron 3343; Instron, Norwood, MA, USA) that was interfaced with a personal computer. Tissue was kept moist before the tensile test to mimic in vivo conditions and avoid sample desiccation, as detailed above. A sample was clamped to the tensiometer using mechanical grips, which moved at

Table 1 Laser parameters for photochemical tissue bonding (mean \pm maximum error)

\begin{tabular}{lcccc}
\hline Area $\left(\mathrm{mm}^{2}\right)$ & Power $(\mathrm{mW})$ & Time $(\mathrm{s})$ & Fluence $\left(\mathrm{J} \mathrm{cm}^{-2}\right)$ & $I\left(\mathrm{~W} \mathrm{~cm}^{-2}\right)$ \\
\hline $60 \pm 5$ & $180 \pm 5$ & $365 \pm 5$ & $110 \pm 10$ & $\sim 0.9$ \\
\hline
\end{tabular}

Area, surface area of the rose adhesive; fluence, laser fluence; I, estimated irradiance; power, laser power; time, irradiation time. a rate of $22 \mathrm{~mm} \mathrm{~min}{ }^{-1}$ until the two tissue stumps separated. The maximum load at which the stumps separated (80\% load drop) was recorded and the repair strength estimated by dividing the max load to the surface area of the adhesive. $^{21}$

\section{Pressure test}

The chitosan adhesive was also used to repair a section of intestine that had a $3 \mathrm{~mm}$ hole in the middle. The adhesive $\left(1 \times 1 \mathrm{~cm}^{2}\right)$ was laser bonded to the intestine to seal the hole, as described above. The intestine was secured to tubing at both ends with plastic cable ties. The tubing at one end was connected to a calibrated transducer (SP844; MEMSCAP, Skoppum, Norway) that was coupled to an infusion pump (NE-4000 Syringe Pump; Adelab Scientific, Thebarton, SA, Australia) and an operational amplifier (ML221 Bridge Amp; ADInstruments, Thebarton, SA, Australia). The infusion pump injected colored water into the intestine at a rate of $6 \mathrm{~cm} \mathrm{~min}^{-1}$ until the rupture of the adhesive repair was observed and noted as burst pressure. The transducer was interfaced to a DAQ (ML866 PowerLab 4/30; ADInstruments) and a personal computer with recording software (LabChart 7 Pro; ADInstruments) for data storage and analysis.

\section{Contact angle and capillary forces}

The static contact angle of chitosan adhesive films was measured using the sessile drop technique (Rame-hart Instrument Co., Sucassuna, NJ, USA) and the contact angle was determined using DROPimage Standard software analysis.

The nanostructured and flat films were positioned on a translating stage and a single $8 \mu \mathrm{l}$ drop of water was lowered onto them. The system software measured the water contact angles of each side of the drop and computed the mean value for 30 films per group $(n=30)$. To assess the impact of capillary forces, the adhesive films were applied to bisected tissue without laser irradiation as detailed above ('Photochemical Tissue Bonding' section); in one group $\sim 100 \mu \mathrm{l}$ of water was poured over the sample and allowed to soak for $2 \mathrm{~min}$, following the application of the adhesive to tissue. The tensile test was performed after watering in order to ensure the samples were wet ('wet repair'). In another group, the bisected tissue was allowed to dry for 3 min before applying the adhesive and the sample was subsequently tested to assess adhesion strength ('dry repair'). It was noted that tissue could shrink and partially dislodge the adhesive, if it dried for longer period of time. The third group ('moist repair') consisted of tissue that was repaired as detailed in the 'Photochemical Tissue Bonding' section, without any extra wetting or drying steps.

\section{Atomic force microscopy and spectroscopy}

The surface topography of samples with and without nanostructures was characterized using an atomic force microscope (NanoWizard II; JPK Instruments AG, Berlin, Germany). For imaging the microscope was operated in intermittent contact mode in air using silicon cantilevers with a nominal spring constant of $36 \mathrm{~N} \mathrm{~m}^{-1}$ and a resonant frequency of $300 \mathrm{kHz}$ (type ACT; AppNano, Mountain View, CA, USA). The scanning rate was set to $1 \mathrm{~Hz}$ with a pixel resolution of $2048 \times 2048$. Six films were scanned for each group and 12 measurements of the surface roughness were performed over an area of $50 \times 50 \mu \mathrm{m}$ on separate films.

For force spectroscopy, the measurements were undertaken in water to eliminate capillary effects using a silicon cantilever with a nominal spring constant of $0.29 \mathrm{~N} \mathrm{~m}^{-1}$ and a resonant frequency of $15 \mathrm{kHz}$ (type SICON; AppNano). All cantilevers were individually calibrated using the thermal noise method prior to usage. For all measurements the following parameters remained constant: a Z-length of $2.0 \mu \mathrm{m}$, an extend time of $2.0 \mathrm{~s}$, with a relative setpoint of $5 \mathrm{nN}$. Force measurements were performed in 20 random locations over a sample size of $50 \times 50 \mu \mathrm{m}^{2}$ on five different samples $\left(6 \times 10 \mathrm{~mm}^{2}\right)$ of flat or nanostructured films $(n=100)$; the same measures were repeated using two tips for a total of 200 measures in each experimental group. Force measurements were performed on chitosan films that were either preirradiated by the laser, or not, to assess the effect of the laser on the adhesive. 

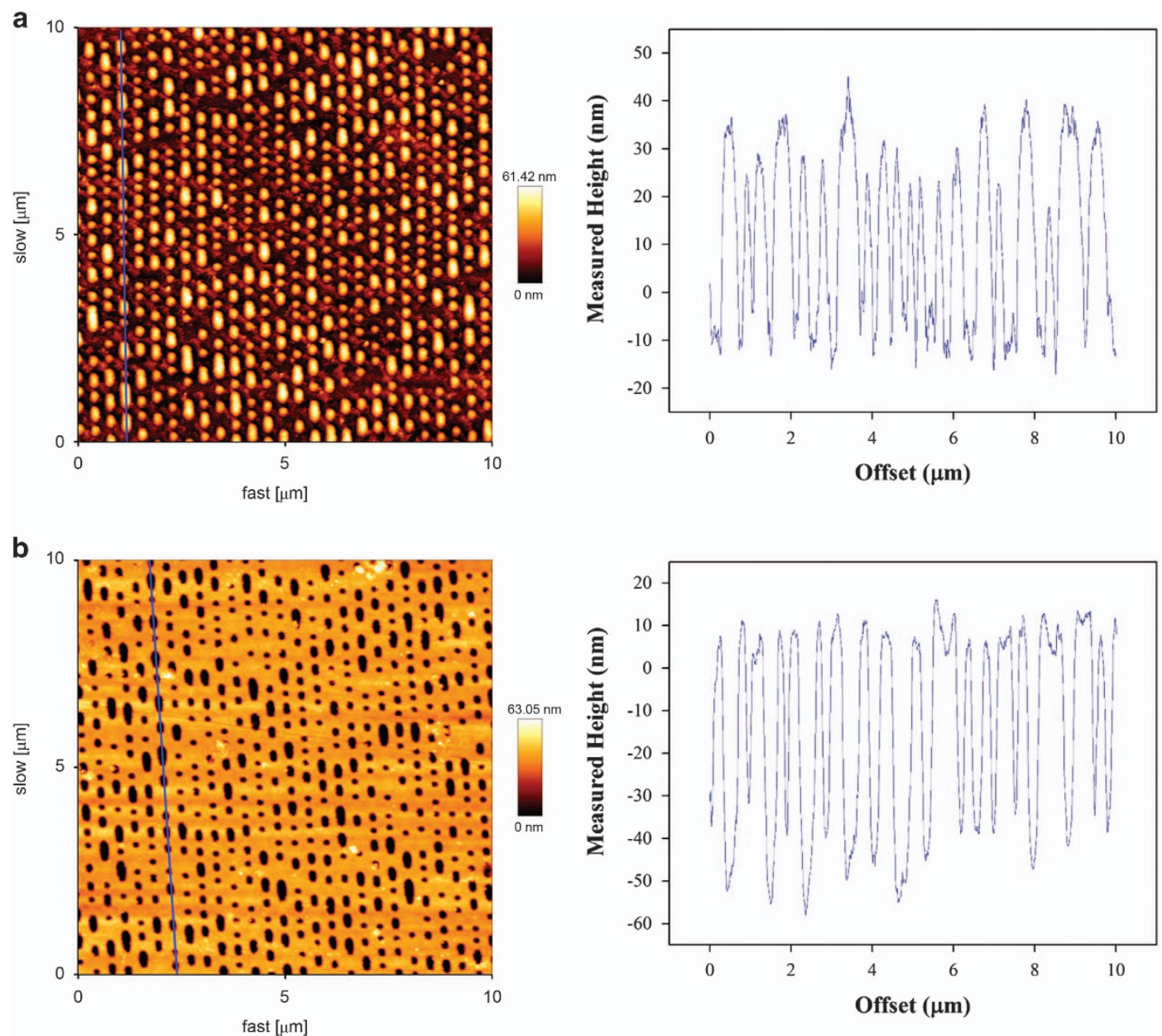

Figure 1 (a) The atomic force microscopy image of the nanostructured surface of the chitosan adhesive film; the nanopillars form a regular pattern over the surface that replicates the polycarbonate master plate depicted in (b). The blue trace shows a typical height profile of the nanopillars that have base length of $~ 100,200,300,400,500$ and $600 \mathrm{~nm}$ and height ranging from 40 to $70 \mathrm{~nm}$; the nanopillars match the axial lengths and depths of the master plate holes.

\section{Scanning electron microscopy}

To visualize the nanostructures on chitosan adhesive films and their bonding to tissue, transverse sections through the intestine and adhesive were fixed in a Karnovsky's solution (2.5\% paraformaldehyde and $2 \%$ glutaraldehyde in $0.1 \mathrm{M}$ phosphate buffer) overnight at $4{ }^{\circ} \mathrm{C}$. Both the adhered tissue and chitosan alone were washed twice with $0.1 \mathrm{M}$ phosphate buffer for $15 \mathrm{~min}$ each. The samples were then dehydrated through an ethanol series $(30,50,70,80,90,95$, and $100 \%$ ) and processed using a critical point dryer (Autosamdri-815; Tousimis, Rockville, MD, USA). The samples were then mounted onto aluminium stubs, coated with $5 \mathrm{~nm}$ platinum (Leica EM ACE600; Leica Microsystem, North Ryde, NSW, Australia) and analyzed at $5 \mathrm{keV}$ on field emission scanning electron microscopy (FEI Nova NanoSEM 450; FEI Company, Hillsboro, OR, USA).

\section{Statistics}

Data values were analyzed using two-way analysis of variance (ANOVA) followed by the Bonferroni post-test or two tails $t$-test at a significant level of 0.05 ; values are expressed as mean \pm s.d.

\section{RESULTS AND DISCUSSION}

The adhesive casted on the polycarbonate master plate replicated nanostructures with high fidelity (Figures $1 \mathrm{a}$ and $\mathrm{b}$ ). The pillars had a height of $62 \pm 18 \mathrm{~nm}(n=72)$ and their shape was approximately hemi-ellipsoidal with the same dimensions of the master-plate elliptic holes (Figures 2 and 3). The master plate had intentional holes with various sizes, ranging from 100 to $600 \mathrm{~nm}$, to demonstrate that the dry-cast technique could reproduce adhesive nanopillars of different dimensions. Some defects were also visible in $\sim 10 \%$ of the pillars on the adhesive that had a surface roughness of $14 \pm 1 \mathrm{~nm}$. The flat adhesive had a roughness of $3 \pm 1 \mathrm{~nm}$ and lacked regular nanostructures as only sporadic peaks $(5-10 \mathrm{~nm})$ appeared on the surface, which were due to random defects on the PMMA master plate.

The water contact angles of the nanopatterned and flat adhesives were $95.3 \pm 3.4^{\circ}$ and $90.0 \pm 6.7^{\circ}$, respectively $(P=0.002$, $t$-test, $n=30)$; these results are similar to previously reported values $\left(89 \pm 6^{\circ}\right)$ of 'flat' films based on chitosan. ${ }^{24,25}$ The Wenzel equation relates the contact angles of the flat and nanopatterned adhesives and is in very good agreement with our data:

$$
\cos \theta^{*}=r \cos \theta \text { (Wenzel equation) }
$$

where $\theta^{\star}$ is the contact angle of the nanopatterned adhesive (unknown variable), $\theta=90^{\circ}$ is the contact angle of the flat adhesive and $r \sim 1.7$ is the surface area ratio between the nanopatterned and flat adhesives. $\theta^{\star}$ is thus equal to $90^{\circ}$ and this calculated angle deviates $\sim 6 \%$ from the measured value. The validity of this equation strongly suggests that the nanopatterned and flat adhesives are not affected by the substrates used for their fabrication (Figure 4). 
a
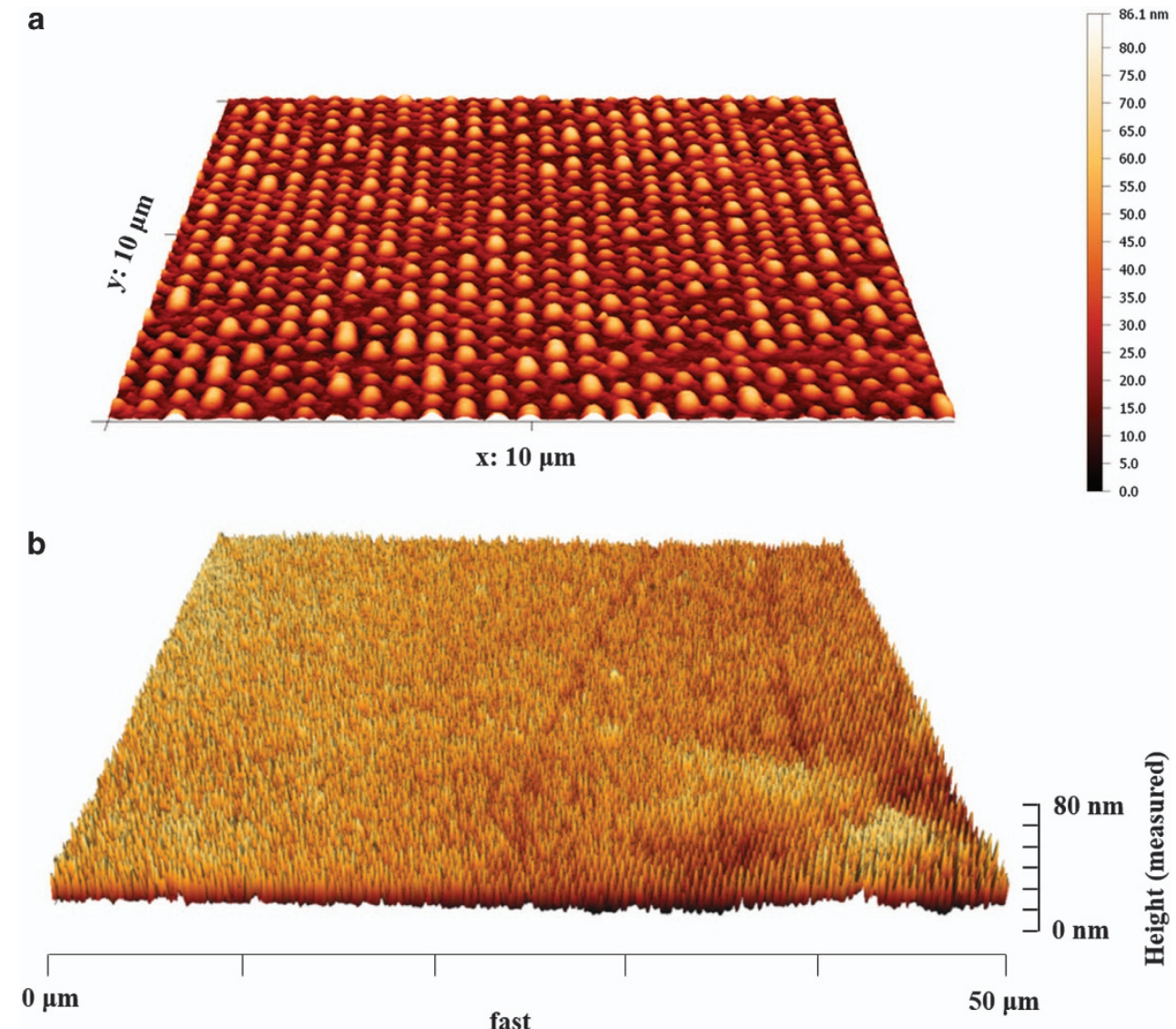

Figure 2 (a) Three-dimensional image of the nanostructured adhesive on a $10 \times 10 \mu \mathrm{m}$ section; (b) the regular pattern of nanopillars is maintained at a larger scale with a small percentage of defects. The $z$-axis scale has been increased to highlight pillar defects.

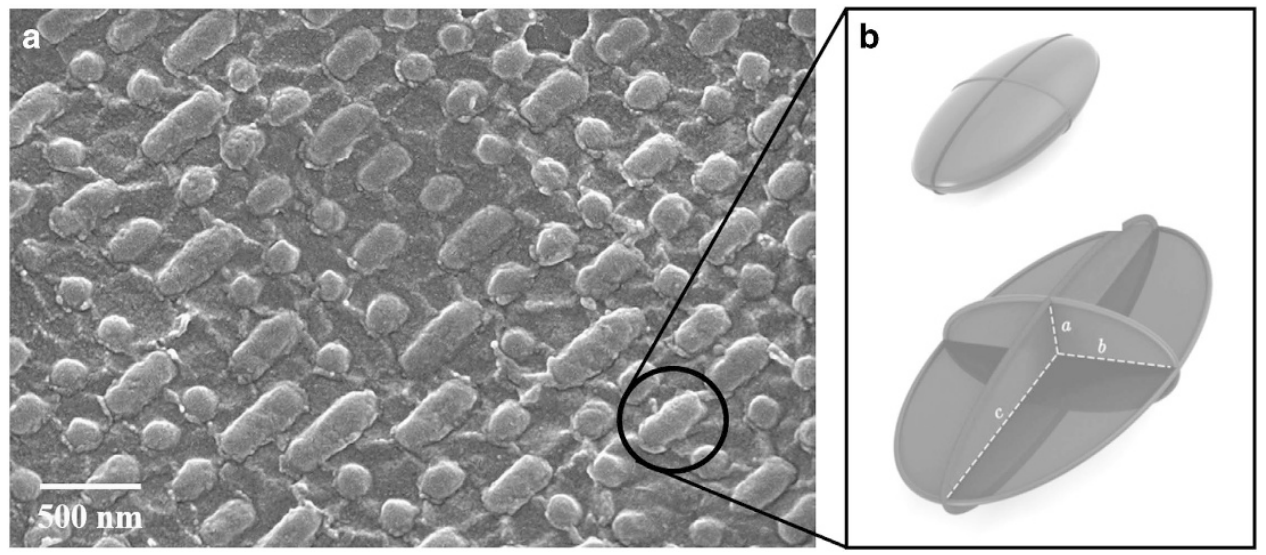

Figure 3 (a) Scanning electron microscopy image of the chitosan adhesive surface; the nanostructures are regularly patterned replicating the master-plate elliptic holes. The pillars resemble hemi-ellipsoids (b) $\sim 62 \mathrm{~nm}$ tall (semiaxis a) with axial lengths $(2 b, 2 c)$ of $\sim 100 \times 100, \sim 100 \times 200, \sim 100 \times 300$, $\sim 100 \times 400, \sim 100 \times 500$ and $\sim 100 \times 600 \mathrm{~nm}$. The nanostructured adhesive has a larger surface area than the flat adhesive due to the lateral surface of the hemi-ellipsoids. The ratio between the lateral surface of the average size pillar and the pillar elliptic base area is $\sim 1.7$, thus the extra surface area can be estimated as $1.7-1=0.7$ or $70 \%$ of the flat adhesive area.

The contact angle of the nanopatterned adhesive is slightly hydrophobic $\left(\theta^{\star}=95.3^{\circ}\right)$ and the water meniscus is convex; the pressure inside the liquid is thus higher than the pressure outside the liquid, causing an outward force that appears to repel the adhesive from tissue. The contact angle of the flat adhesive $\left(\theta=90^{\circ}\right)$ makes the water meniscus flat and thus no pressure is exerted on the adhesive. Capillary forces that are acting on two closely spaced chitosan adhesives are thus either repulsive or null for nanopatterned and flat surfaces, respectively. These forces are in first approximation proportional to the cosine of the contact angle $\left(p \propto \cos \theta^{\star}\right)$ for a fixed thickness of water layer. ${ }^{26}$ When the adhesive is applied on tissue, capillary action occurs between two dissimilar surfaces and the repulsive effect of the nanopatterned adhesive is diminished because of the hydrophilic nature of tissue, ${ }^{27}$ as previously reported. ${ }^{28}$ 
a

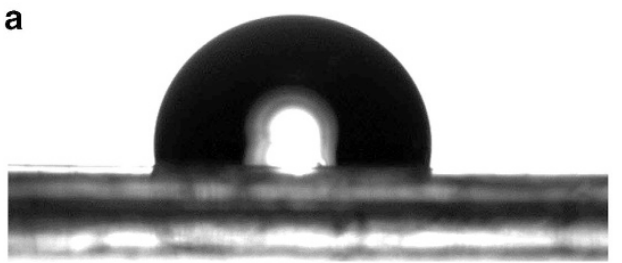

b

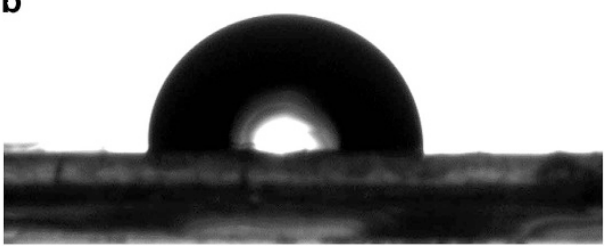

Figure 4 The water contact angle of the nanopatterned adhesive (a) is slightly hydrophobic $\left(\sim 95^{\circ}\right)$ and the flat adhesive has a contact angle of $\sim 90^{\circ}$ (b).

a

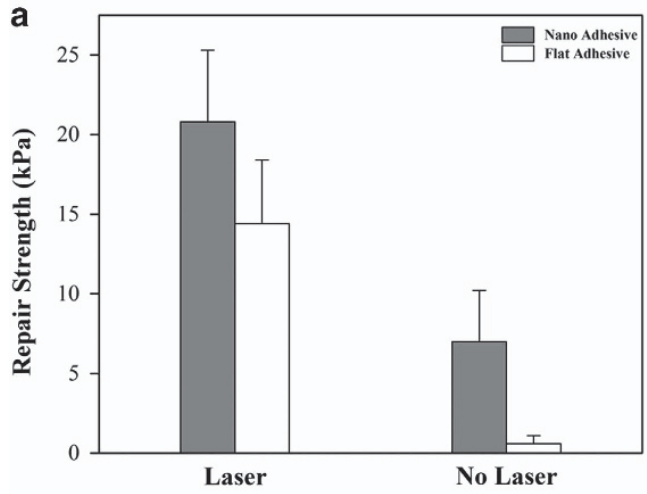

b

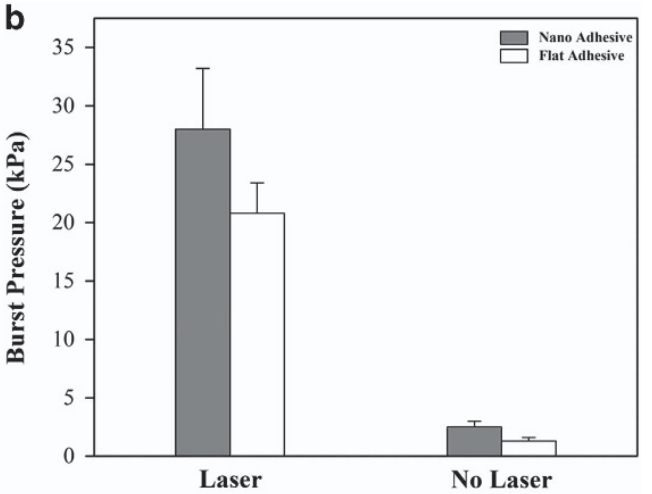

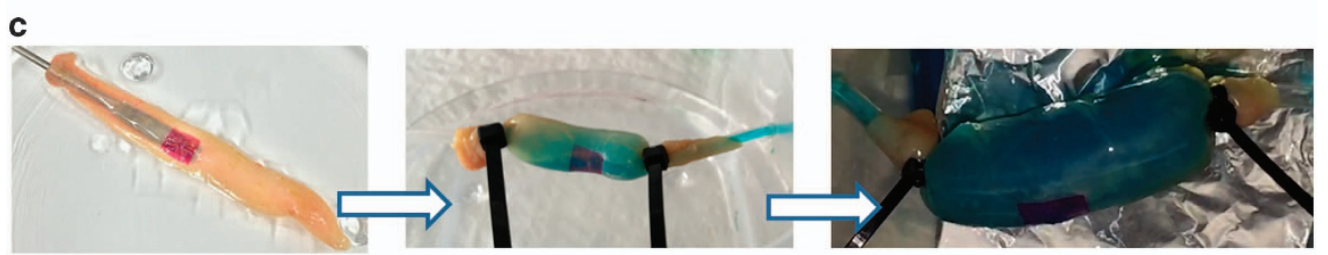

Figure 5 (a) Column chart of the repair strength; the repair strength of the nanostructured adhesive was 11 times stronger than the flat adhesive, when no laser irradiation was applied $(7.0 \pm 3.2$ and $0.6 \pm 0.5 \mathrm{kPa}$, respectively). The nanostructured adhesive was also $~ 30 \%$ stronger than the 'flat' adhesive when laser-activated $(20.8 \pm 4.5$ and $14.4 \pm 4.0 \mathrm{kPa}$, respectively; $P<0.001$, two-way ANOVA, $n=28)$. (b) Column chart of the pressure test; the lasered nanostructured adhesive withstand the highest pressure before bursting $(28.0 \pm 5.2 \mathrm{kPa})$ and was $26 \%$ higher than the flat adhesive $(20.8 \pm 2.6 \mathrm{kPa}$; $P<0.001$, two-way ANOVA, $n=15$ ). The burst pressure was significantly lower when the laser did not bond the adhesive to tissue with or without nanopillars $(\sim 2 \mathrm{kPa})$. (c) Digital photos to exhibit a visual demonstration of the build-up of pressure.

The nanostructured adhesive bonded much stronger to tissue than the flat adhesive (two-way ANOVA, $P<0.001$, Bonferroni post-test, $n=28$ ): without laser irradiation the bonding strengths were $7.0 \pm 3.2$ and $0.6 \pm 0.5 \mathrm{kPa}$, respectively (Figure $5 \mathrm{a}$ ). In this instance, van der Waals and electrostatic forces likely acted between nanopillars and tissue drastically increasing the adhesion. Chitosan films without nano patterns are well known to bond weakly to tissue and mucosae, as previously reported; ${ }^{17,29,30}$ in our case the initial adhesion of the flat adhesive to intestine was $\sim 0.6 \mathrm{kPa}$ before irradiation and electrostatic forces are likely responsible for this weak bonding.

To substantiate the hypothesis that electrostatic and van der Waals forces are more influential than capillary forces, a separate experiment was conducted to assess the impact of moisture on tissue bonding without laser irradiation (Figure 6). The adhesive strength of the nanopatterned and flat adhesives dropped significantly in wet environment $(\sim 1.0 \mathrm{kPa})$ while it increased remarkably when tissue was allowed to dry for $3 \mathrm{~min}(\sim 7.9$ and $\sim 2.6 \mathrm{kPa}$, respectively). These results suggest that attractive electrostatic and van der Waals forces acted on the adhesives and were more predominant than capillary forces, in agreement with the contact angle measurements. The adhesion strength of the flat adhesive over wet and moist tissue did not change significantly $(\sim 1.0 \mathrm{kPa})$, reinforcing the hypothesis that capillary forces were not influential. The nanopatterned adhesive had by contrast a significant increment in adhesion strength $(\sim 6.3 \mathrm{kPa})$ on moist tissue when compared with wet tissue $(\sim 1.1 \mathrm{kPa})$ that could result from van der Waals forces, or equivalently, from the close proximity of the nano pillars to tissue. On 3-min dried tissue, electrostatic forces were likely responsible for the bonding increase of the flat adhesives with respect to moist tissue, while the large increment in adhesion for the nanopatterned adhesive was probably due to the combination of van der Waals forces and electrostatic forces, the latter arising from the flat portion of the nanostructured adhesive (Figure 6). The adhesive is positively charged due to the presence of the protonated amino group in the D-glucosamine residues of the chitosan. Our results strongly suggest that the flat adhesive does not contact tissue as effectively as the nanopatterned adhesive, which is more efficient in binding to tissue. The results are summarized in Table 2.

The nanostructured adhesive had a larger surface area in contact to tissue than the flat adhesive that may be ascribed to the surface of the nanopillars; this surface increment can be heuristically estimated around $70 \%$ if we consider the ratio between the pillar lateral surface and base area. In this case, the hemi-ellipsoid area of the pillar is $S_{\mathrm{H}} \sim 2 \pi\left[\left(a^{p} b^{p}+a^{p} c^{p}+b^{p} c^{p}\right) / 3\right]^{1 / p}$, the ellipse area is $S_{\mathrm{E}} \sim \pi a b$ and $S_{\mathrm{H}} / S_{\mathrm{E}} \sim 1.7$, where $a=60 \mathrm{~nm}, b=150 \mathrm{~nm}$ and $c=62 \mathrm{~nm}$ are the average values of the ellipsoid semiaxes, and $p=1.6075$ accordingly to Thomsen's formula (Figure 3). ${ }^{31}$ The bonding strength of the nanostructured adhesive should therefore have increased 


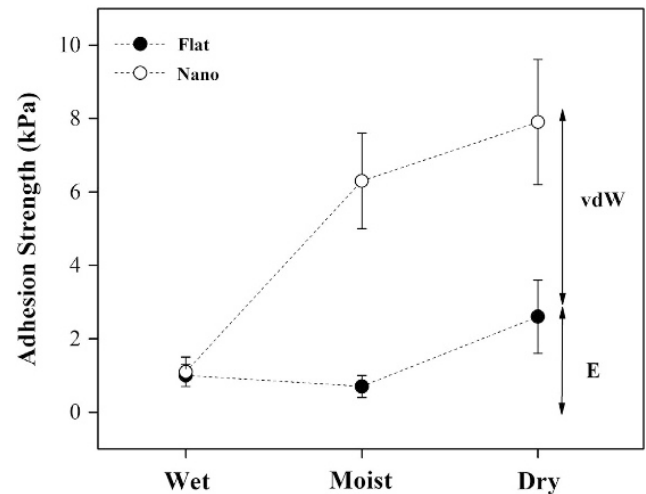

Figure 6 Bonding strength of the nanopatterned and flat adhesives that were applied on wet, moist and dry tissue, and without laser irradiation. No significant bonding variation was recorded during wet and moist repair with flat adhesives, indicating no influence from capillary forces. The bonding strength of the nanopatterned adhesive greatly increased on moist tissue because of van der Waals forces. Electrostatic and van der Waals forces appeared to be prominent as tissue became dryer. The adhesion strengths due to electrostatic (E) and van der Waals ( $v d W$ ) forces are indicated in the plot by arrows $(\sim 2.6$ and $\sim 5.3 \mathrm{kPa}$, respectively).

Table 2 Bonding strengths of chitosan adhesives applied on tissue without laser irradiation and under different hydration conditions

\begin{tabular}{llll}
\hline & Wet $(\mathrm{kPa})$ & Moist $(\mathrm{kPa})$ & Dry $(\mathrm{kPa})$ \\
\hline Nano adhesive & $1.1 \pm 0.4$ & $6.3 \pm 1.3^{*}$ & $7.9 \pm 1.7^{*}$ \\
Flat adhesive & $1.0 \pm 0.3$ & $0.7 \pm 0.3$ & $2.6 \pm 1.0^{*}$ \\
\hline
\end{tabular}

Wet, $\sim 100 \mu$ l of water was applied on samples; moist, $\sim 40 \mu \mathrm{l}$ of water was applied on samples; dry, tissue was allowed to dry for $3 \mathrm{~min}$ (please refer to text for more details).

$* P<0.001$, two-way ANOVA, Bonferroni post-test, $n=15$.

proportionally to the extra surface area $(\sim 70 \%)$ while our results showed a much greater increment, an indication that van der Waals forces are contributing to the adhesion strength. ${ }^{1,2}$

When the laser is applied, the bonding strength of the nanostructured adhesive is $30 \%$ stronger $(20.8 \pm 4.5 \mathrm{kPa})$ than the flat adhesive $(14.4 \pm 4.0 \mathrm{kPa} ; P<0.001$, Bonferroni post-test, two-way ANOVA, $n=28$ ). We may interpret this increase in bonding strength and adhesion primarily to the photocrosslinking between chitosan and tissue; in particular, the known ability of rose bengal to produce singlet oxygen upon green light irradiation facilitates the crosslinking between collagen and chitosan via amino groups. ${ }^{32-34}$ Photochemical tissue bonding is enhanced in the case of the nanostructured adhesive by the regularly patterned pillars and increased surface area that can promote more contacts with conforming tissue (Figure 7). Further investigations involving electron spin resonance, spin trapping, solid state nuclear magnetic resonance and infrared spectroscopy are needed to elucidate the molecular mechanism underlying the bonding between chitosan films and tissue using green lasers. ${ }^{35}$

The outcome of the pressure test is in agreement with the above results, as seen in Figures $5 b$ and $c$; the burst pressure of the nanostructured adhesive $(28.0 \pm 5.2 \mathrm{kPa})$ was $26 \%$ higher than the flat adhesive when laser irradiated $(20.8 \pm 2.6 \mathrm{kPa} ; P<0.001$, Bonferroni post-test, two-way ANOVA, $n=15)$. There was no statistical difference between the burst pressure of the nanostructured and flat adhesives without laser activation $(2.2 \pm 0.5$ and $1.3 \pm 0.3 \mathrm{kPa}$, respectively; $P>0.05$, Bonferroni post-test, two-way ANOVA, $n=15$ ). In this case, the perpendicular force exerted by the water on the adhesive effectively detached the nanopillars from tissue. The poor

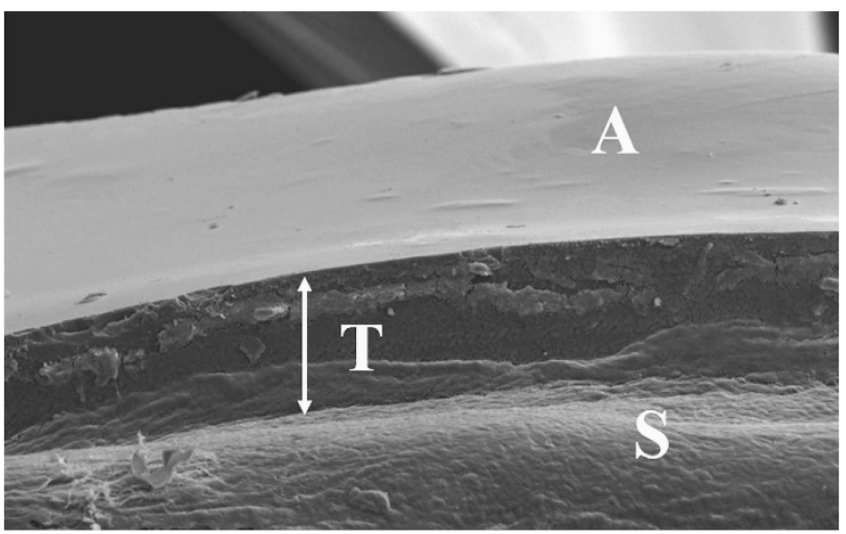

Figure 7 Scanning electron microscopy image of the nanostructured adhesive (A) bonded to intestine serosa (S); the adhesive has a regular thickness $(T)$ and conforms to tissue following its curved shape.

adhesion of the nanostructured adhesive under pressure was also influenced by the presence of water that weakened electrostatic and van der Waals forces between tissue and nanopillars as the Hamaker constant is lower in water than in air. ${ }^{4}$ The (non-irradiated) nanostructured adhesive behaved very differently when pulled tangentially to the tissue surface; in this instance the adhesion strength increased $\sim 11$ times with respect to the flat adhesive. This outcome resembles the contact adhesion mechanism of the gecko, whereby the gecko's foot is dragged across a surface increasing the number of spatulae in contact and maximizing the adhesion force. ${ }^{1}$ In our case, we can infer that the tangential pull of the tensiometer facilitated and improved the contact of nanopillars to tissue.

During burst pressure and tensile tests, the adhesive always detached from tissue without breaking (surface failure) but in a few cases where four flat irradiated samples broke into two parts under tension (cohesive failure).

The force spectroscopy results are displayed in Figure 8 . These results confirm that the nanopatterned adhesive (Figure 8a) exerts stronger attraction than the flat adhesive (Figure $8 \mathrm{~b}$ ) on the cantilever tip without laser irradiation $(-5.5 \pm 3.1$ and $-2.4 \pm 0.7 \mathrm{nN}$, respectively; $P<0.01$, two-way ANOVA, Bonferroni post-test). A factor that can influence the stronger attraction of the atomic force microscopy cantilever to the nanopatterned adhesive is due to the fact that the tip can land either on the semihellipsoidal pillar or on the flat surface. When the tip lands on the top of the pillar, it experiences a force that is similar to the force measured on the flat adhesive as the tip radius of curvature is much smaller $(\sim 6 \mathrm{~nm})$ than the dimensions of the top of the pillar (100-600 nm). However, the tip can also lend on the pillar edge and in this case more attractive forces may arise from the contact between the tip shaft (length $=15 \mu \mathrm{m}$ ) and the pillar edge.

The energy required to completely detach the tip from the nanopatterned adhesive, which is the area under the blue curve, was more than double the adhesion energy of the flat adhesive $\left(5.1 \pm 3.8 \times 10^{-16}\right.$ and $2.2 \pm 0.7 \times 10^{-16} \mathrm{~J}$, respectively; $P<0.05$, twoway ANOVA, Bonferroni post-test). The blue curve shows that upon retraction from the chitosan surface there is an initial direct-surface adhesion, where the maximum force is taken, due to detachment of most of the chitosan from the cantilever tip. Following this, several chitosan polymer chains remain tethered between the cantilever and surface, and are subsequently 'stretched' as the cantilever continues to retract from the surface. ${ }^{36}$ Stretching of the polymer chains results in the observation of multiple force peaks (ruptures) that extend to 
a

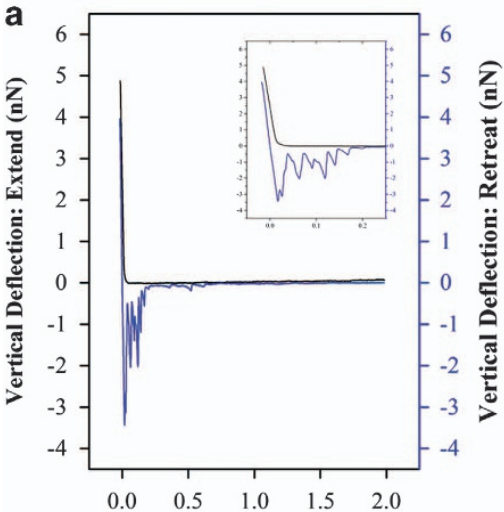

Tip-Sample Separation $(\mu \mathrm{m})$ b

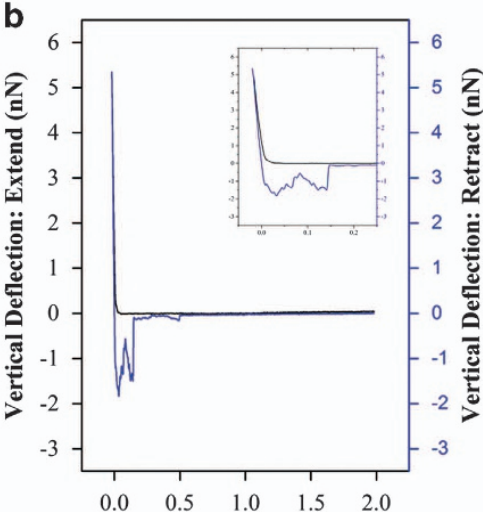

Tip-Sample Seperation $(\mu \mathrm{m})$

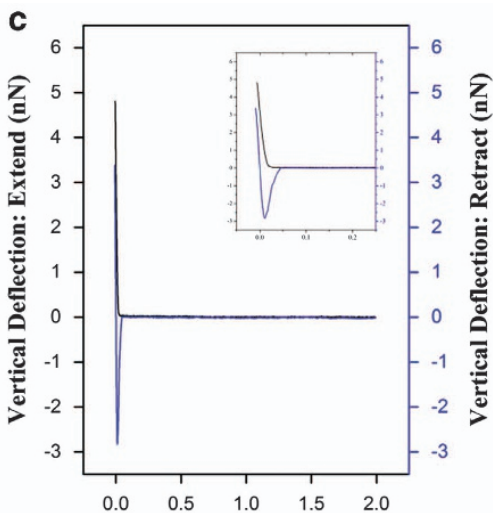

Tip-Sample Seperation $(\mu \mathrm{m})$

Figure 8 Typical force spectroscopy scans for the (non-irradiated) nanostructured adhesive (a) and flat (irradiated) adhesives (b). The microscope operated in water to eliminate capillary effects. The blue curve shows that the cantilever tip experienced an attractive force when retracted due to detachment of most of the chitosan from the tip. A number of chitosan polymer chains remain nevertheless tethered between the tip and surface, and are subsequently 'stretched' when the cantilever continues to retract from the surface, resulting in multiple force peaks (insets). No multiple peaks are observed when the adhesive is crosslinked by cyanoacrylate glue before the test (c). The energy required to completely detach the tip from the adhesive is the area under the blue curve and the nanopatterned adhesive has higher adhesion energy than the flat adhesive. Insets are expansion of the data between 0 and $0.25 \mu \mathrm{m}$.

distances up to a micron. The multiple ruptures are likely due to the sequential breaking of bonds, including further detachment of chitosan polymer chains from the cantilever but also inter- and intramolecular bonds between and within the polymer chains. No ruptures were observed when force spectroscopy was performed on the nanopatterned and flat adhesives that were crosslinked with commercial cyanoacrylate glue before testing (Figure 8c). It is evident that the adhesion is related to the binding of chitosan polymer chains and an increase in the contact area between the probe and the adhesive surface can lead to binding of more chitosan chains, thereby increasing adhesion strength and energy. The binding may occur through electrostatic interactions as the chitosan adhesive is positively charged while the silica probe has a negative charge. Van der Waals and electrostatic interactions are weak in liquid and for this reason the atomic force microscopy force curves showed no significant attractive force upon approach (black curve) but only a repulsive interaction (Figure 8).

The force profiles are similar to that described previously for natural materials ${ }^{36}$ where the stretching of polymer chains, and sequential breaking of bonds as they are placed under a tensile stress, increases the adhesion energy of the interaction. Thus, the nanopatterned chitosan adhesive effectively combines two mechanisms for tissue bonding: one based on a nanopatterned surface that increases the adhesion strength (via increase in number of van der Waals interactions) over short distances and another based on an interaction with a polymer surface that involves the elastic, unbinding forces of polymer chains that occur over much longer distances and increase the overall adhesion energy. Based on these mechanisms, the nanostructured chitosan system has increased adhesion strength combined with increased toughness (adhesion energy).

When the adhesive is preirradiated by the laser, the force curves have also multiple peaks and are very similar to the curves of the non-irradiated adhesives; the average values of force and energy of the nanostructured adhesive are higher than the flat adhesive although they are not statistically different (Table 3 ). In this case we notice that the standard deviation of the nanostructured adhesive is much larger than that of the flat adhesive, obscuring statistical significance. The large standard deviation is due to the cantilever tip landing either on
Table 3 Summary of the atomic force spectroscopy results: the maximum force $(\mathrm{nN})$ and adhesion energy $(\mathrm{J})$ are reported for the nanostructured and flat adhesives, which have been preirradiated or not

\begin{tabular}{lr}
\hline Flat adhesive & Nanoadhesive \\
\hline No laser & \\
$-2.4 \pm 0.7 \mathrm{nN}$ & $-5.5 \pm 3.1 \mathrm{nN}^{*}$ \\
$2.2 \pm 0.7 \times 10^{-16} \mathrm{~J}$ & $5.1 \pm 3.8 \times 10^{-16} \mathrm{~J}^{*}$ \\
& \\
Preirradiated & \\
$-2.3 \pm 0.7 \mathrm{nN}$ & $-3.8 \pm 1.6 \mathrm{nN}$ \\
$2.5 \pm 0.6 \times 10^{-16} \mathrm{~J}$ & $4.7 \pm 3.5 \times 10^{-16} \mathrm{~J}$ \\
\hline
\end{tabular}

${ }^{*} P<0.01$, two-way ANOVA, Bonferroni post-test.

nanopillars (stronger attraction) or on the flat surface of the adhesive. These results indicate that elastic and unbinding forces of polymer chains still occur in the irradiated adhesive, enhancing the adhesive toughness. A previous study showed that the Young's modulus of the rose bengal-chitosan adhesive is unchanged when it is laser irradiated, ${ }^{21}$ suggesting that the laser does not crosslink the bulk of the adhesive; our results also point out that the laser irradiation does not noticeable alter the adhesive rigidity.

The nanostructured chitosan adhesive is a major improvement to previously reported Gecko-inspired adhesives for tissue repair because it does not require an extra layer of polymeric coating on nanopillars to bond strongly to tissue under wet physiological conditions $(\sim 21 \mathrm{kPa})$. Lee et al. ${ }^{6}$ for example, fabricated a wet and reversible adhesive using a nanopillared substrate made of non-degradable polydimethylsiloxane that was coated with a mussel-adhesiveprotein-mimetic polymer. Mahdavi et al. ${ }^{7}$ used a similar approach by including a coating of oxidized dextran on poly(glycerol sebacate acrylate) pillars, ranging from $100 \mathrm{~nm}$ to $1 \mu \mathrm{m}$ in width and $0.8-3.0 \mu \mathrm{m}$ in height, to promote crosslinking with the tissue and ensuring a non-reversible chemical bond; the adhesive achieved a maximum separation force ranging between 29 and $48 \mathrm{kPa}$ on porcine 
intestinal tissue in vitro. Pereira et al. ${ }^{8}$ developed a micropatterned adhesive $(\sim 25 \mathrm{kPa})$ made from poly( $\varepsilon$-caprolactone $)$ with a spin-coated layer of medical-grade cyanoacrylate adhesive. The adhesive was applied to seal stomach and colon perforation in rats, showing minimal inflammatory response 3 weeks postoperatively. These adhesives require two or three steps for their fabrication while the chitosan adhesive relies on a simple dry-casting technique with obvious advantages. Fernandez et al. ${ }^{37}$ reported the fabrication of chitosan-based scaffolds with micrometer patterned pillars. The scaffolds had no tissue adhesive capability and were fabricated in several steps. In particular, the dry casted scaffolds were immersed twice in a solution of sodium hydroxide and rinsed in distilled water to make them insoluble and avoid dissolution when in contact to tissue. These films swelled in water, were thicker $(\sim 170 \mu \mathrm{m})$ and had larger pillars $(\sim 5 \mu \mathrm{m}$ diameter, $\sim 1 \mu \mathrm{m}$ tall $)$ than the nanostructured adhesive described in this report, which is inherently insoluble. About $10 \%$ of the adhesive nanopillars presented some defects that can be corrected if the dry-casting technique is optimized, for example, exploring different materials for the master plate such as polydimethylsiloxane or Perspex. ${ }^{6,37}$ The flat chitosan adhesive has been successfully used to repair rat median nerves in vivo, ${ }^{18,19}$ showing repair strength comparable to sutures. Myelinated axons regenerated equally well either in rats operated with the flat chitosan adhesives or in sutured nerves; the laser-adhesive technique was nonetheless superior and less invasive than suturing when the functional recovery of nerves was tested 3 months postoperatively. ${ }^{19}$ Of relevance is the fact that the nanoscale modification of the flat chitosan adhesive did not alter its chemical composition and thus its excellent biocompatibility and biodegradability. ${ }^{38}$ In this respect, a recent study has highlighted that the degradation of the flat chitosan adhesives can be modulated if a physiological amount of lysozyme is added during the fabrication process of the adhesive. The lysozyme loaded adhesive degraded $20 \%$ after 4 weeks implantation into biocompatible monomers and oligomers of glucosamine and $N$-acetyl-glucosamine. ${ }^{39}$

In summary, this study describes a monolayer nanostructured film, which is biocompatible and bonds strongly to tissue upon laser activation. By probing the nanoscale interactions and forces with an atomic force microscope, the study has given insight into the molecular mechanisms of the chitosan adhesive as it is placed under stress. The adhesive fabrication requires a simple dry-casting technique that considerably simplifies the more convoluted procedures currently used to manufacture gecko-inspired polymeric films.

\section{CONFLICT OF INTEREST}

The authors declare no conflict of interest.

\section{ACKNOWLEDGEMENTS}

DM was supported by Marie Curie actions FP7 through the Intra-European Marie Curie Fellowship 'MultiFun CP' under grant agreement no. 328897. SJF was supported by an Australian Post-Graduate Award.

1 Autumn, K., Liang, Y. A., Hsieh, S. T., Zesch, W., Chan, W. P., Kenny, T. W. Fearing, R. \& Full, R. J. Adhesive force of a single gecko foot-hair. Nature 405, 681-685 (2000).

2 London, F. The general theory of molecular forces. Trans. Faraday Soc. 33, 8b-26 (1937).

3 Qu, L., Dai, L., Stone, M., Xia, Z. \& Wang, Z. L. Carbon nanotube arrays with strong shear binding-on and easy normal lifting-off. Science 322, 238-242 (2008).

4 Bergström, L. Hamaker constants of inorganic materials. Adv. Colloid Interf. Sci. 70 , 125-169 (1997).

5 Vajpayee, S., Jagota, A. \& Hui, C.-Y. Adhesion of a fibrillar interface on wet and rough surfaces. J. Adhes. 86, 39-61 (2010).
6 Lee, H., Lee, B. P. \& Messersmith, P. B. A reversible wet/dry adhesive inspired by mussels and geckos. Nature 448, 338-341 (2007).

7 Mahdavi, A., Ferreira, L., Sundback, C., Nichol, J. W., Chan, E. P., Carter, D. J., Bettinger, C. J., Patanavanich, S., Chignozha, L., Ben-Joseph, E., Galakatos, A., Pryor, H., Pomerantseva, I., Masiakos, P. T., Faquin, W., Zumbuehl, A., Hong, S., Borenstein, J., Vacanti, J., Langer, R. \& Karp, J. M. A biodegradable and biocompatible gecko-inspired tissue adhesive. Proc. Natl. Acad. Sci. USA 105, 2307-2312 (2008)

8 Pereira, M. J., Sundback, C. A., Lang, N., Cho, W. K., Pomerantseva, I., Ouyang, B., Tao, S. L., McHugh, K., Mwizerwa, O., Vemula, P. K., Mochel, M. C., Carter, D. J., Borenstein, J. T., Langer, R., Ferreira, L. S., Karp, J. M. \& Masiakos, P. T. Combined surface micropatterning and reactive chemistry maximizes tissue adhesion with minimal inflammation. Adv. Healthc. Mater. 3, 565-571 (2014).

9 Gill, I. S., Desai, M. M., Kaouk, J. H., Meraney, A. M., Murphy, D. P., Sung, G. T. \& Novick, A. C. Laparoscopic partial nephrectomy for renal tumor: duplicating open surgical techniques. J. Urol. 167, 469-476 (2002).

10 Ogan, K., Jacomides, L., Saboorian, H., Koeneman, K., Li, Y., Napper, C., Hoopman, J., Pearle, M. S. \& Cadeddu, J. A. Sutureless laparoscopic heminephrectomy using laser tissue soldering. J. Endourol. 17, 295-300 (2003).

11 Murray, K. D., Ho, C.-H., Hsia, J.-Y.J. \& Little, A. G. The influence of pulmonary staple line reinforcement on air leaks. CHEST 122, 2146 (2002).

12 Downey, D. M., Harre, J. G. \& Pratt, J. W. Functional comparison of staple line reinforcements in lung resection. Ann. Thorac. Surg. 82, 1880-1883 (2006).

13 Lumsden, A. B. \& Heyman, E. R. Prospective randomized study evaluating an absorbable cyanoacrylate for use in vascular reconstructions. J. Vasc. Surg. 44, 1002-1009 (2006)

14 Hida, K., Yamaguchi, S., Seki, T., Yano, S., Akino, M., Terasaka, S., Uchida, T. \& Iwasaki, Y. Nonsuture dural repair using polyglycolic acid mesh and fibrin glue: clinical application to spinal surgery. Surg. Neurol. 65, 136-142 (2006).

15 Cimador, M., Castagnetti, M., Milazzo, M., Sergio, M. \& De Grazia, E. Suture materials: do they affect fistula and stricture rates in flap urethroplasties? Urol. Int. 73, 320-324 (2004)

16 Alfieri, S., Carriero, C., Caprino, P., Di Giorgio, A., Sgadari, A., Crucitti, F. \& Doglietto, G. B. Avoiding early postoperative complications in liver surgery. A multivariate analysis of 254 patients consecutively observed. Dig. Liver Dis. 33, 341-346 (2001).

17 Lauto, A., Mawad, D., Barton, M., Gupta, A., Piller, S. C. \& Hook, J. Photochemical tissue bonding with chitosan adhesive films. Biomed. Eng. Online 9, 47 (2010).

18 Barton, M., Morley, J. W., Stoodley, M. A., Ng, K. S., Piller, S. C., Duong, H., Mawad, D., Mahns, D. A. \& Lauto, A. Laser-activated adhesive films for sutureless median nerve anastomosis. J. Biophotonics 6, 938-949 (2013).

19 Barton, M. J., Morley, J. W., Stoodley, M. A., Shaikh, S., Mahns, D. A. \& Lauto, A. Long term recovery of median nerve repair using laser-activated chitosan adhesive films. J. Biophotonics 8, 196-207 (2015).

20 Lauto, A., Stoodley, M., Barton, M., Morley, J. W., Mahns, D. A., Longo, L. \& Mawad, D. Fabrication and application of rose bengal-chitosan films in laser tissue repair. J. Vis. Exp 68 (2012).

21 Barton, M. J., Morley, J. W., Mahns, D. A., Mawad, D., Wuhrer, R., Fania, D., Frost, S. J., Loebbe, C. \& Lauto, A. Tissue repair strength using chitosan adhesives with different physical-chemical characteristics. J. Biophotonics 7 , 948-955 (2014).

22 del Campo, A. \& Arzt, E. Fabrication approaches for generating complex micro-and nanopatterns on polymeric surfaces. Chem. Rev. 108, 911-945 (2008).

23 Zhiltsova, T. V., Oliveira, M. S. A. \& Ferreira, J. A. Integral approach for production of thermoplastics microparts by injection moulding. J. Mater. Sci. 48, 81-94 (2013).

24 Rivero, S., García, M. A. \& Pinotti, A. Physical and chemical treatments on chitosan matrix to modify film properties and kinetics of biodegradation. J. Mater. Phys. Chem. 1, 51-57 (2013).

25 Croisier, F. \& Jérôme, C. Chitosan-based biomaterials for tissue engineering. Eur. Polym. J. 49, 780-792 (2013).

26 Landau, L. D., Akhiezer, A. I., Lifshitz, E. M. in General Physics Mechanics and Molecular Physics 1st edn (eds Sykes J. B., Petford A. D. \& Petford C. L.) Ch. 97, 285-288 (Pergamon Press Ltd Headinton Hill Hall, Oxford, England, 1967).

27 Chung, C., van Hoof, L., Policova, Z., Beharry, S., Sherman, P. M., Neumann, A. W. \& Durie, $P$. Surface hydrophobicity is increased in the ileum and proximal colon of cystic fibrosis mice. Pediatr. Res. 46, 174-178 (1999).

28 Bullard, J. W. \& Garboczi, E. J. Capillary rise between planar surfaces. Phys. Rev. E. Stat. Nonlin. Soft. Matter. Phys. 79, 011604 (2009).

29 Koland, M., Charyulu, R. N., Vijayanarayana, K. \& Prabhu, P. In vitro and in vivo evaluation of chitosan buccal films of ondansetron hydrochloride. Int. J. Pharm. Investig. 1, 164 (2011).

30 Costa, I. D. S. M., Abranches, R. P., Garcia, M. T. J. \& Pierre, M. B. R. Chitosan-based mucoadhesive films containing 5-aminolevulinic acid for buccal cancer's treatment. J. Photochem. Photobiol. B 140, 266-275 (2014).

31 Carbognani, A., Tanga, P., Cellino, A., Delbo, M., Mottola, S. \& Marchese, E. The representation of asteroid shapes: a test for the inversion of Gaia photometry. Planet. Space Sci. 73, 80-85 (2012).

32 Verter, E. E., Gisel, T. E., Yang, P., Johnson, A. J., Redmond, R. W. \& Kochevar, I. E. Light-initiated bonding of amniotic membrane to cornea. Invest. Ophthalmol. Vis. Sci. 52, 9470-9477 (2011). 
33 Verweu, H. \& Steveninck, J. V. Model studies on photodynamic crosslinking. Photochem. Photobiol. 35, 265-267 (1982).

34 Shen, H.-R., Spikes, J. D., Kopečková, P. \& Kopeček, J. Photodynamic crosslinking of proteins II. Photocrosslinking of a model protein-ribonuclease A. J. Photochem. Photobiol. B 35, 213-219 (1996).

35 Sionkowska, A., Wisniewski, M., Skopinska, J., Kennedy, C. J. \& Wess, T. J. Molecular interactions in collagen and chitosan blends. Biomaterials 25, 795-801 (2004).

36 Smith, B. L., Schäffer, T. E., Viani, M., Thompson, J. B., Frederick, N. A., Kindt, J., Belcher, A., Stucky, G. D., Morse, D. E. \& Hansma, P. K. Molecular mechanistic origin of the toughness of natural adhesives, fibres and composites. Nature 399, 761-763 (1999).

37 Fernandez, J. G., Mills, C. A. \& Samitier, J. Complex microstructured 3D surfaces using chitosan biopolymer. Small 5, 614-620 (2009).

38 Muzzarelli, R. A. Biomedical exploitation of chitin and chitosan via mechano-chemical disassembly, electrospinning, dissolution in imidazolium ionic liquids, and supercritical drying. Mar. Drugs 9, 1510-1533 (2011).
39 Mawad, D., Warren, C., Barton, M., Mahns, D., Morley, J., Pham, B. T., Pham, N. T., Kueh, S. \& Lauto, A. Lysozyme depolymerization of photo-activated chitosan adhesive films. Carbohydr Polym. 121, 56-63 (2015).

(c) (i) This work is licensed under a Creative Commons Attribution 4.0 International License. The images or other third party material in this article are included in the article's Creative Commons license, unless indicated otherwise in the credit line; if the material is not included under the Creative Commons license, users will need to obtain permission from the license holder to reproduce the material. To view a copy of this license, visit http:// creativecommons.org/licenses/by/4.0/ 